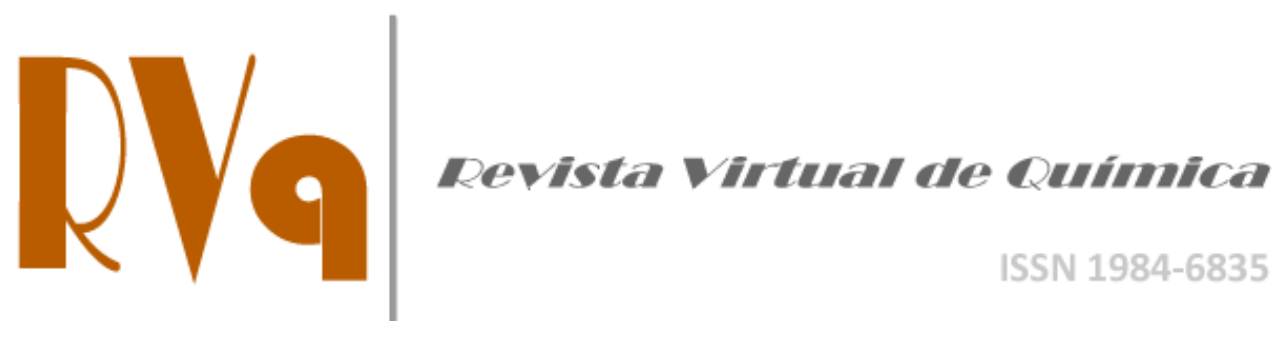

Métodos de Preparação Industrial de Solventes e Reagentes Químicos

2-Diazoacetoacetato de Etila (CAS No. 2009-97-4)

Lima, F. A.*

Rev. Virtual Quim., 2016, 8 (6), 2123-2137. Data de publicação na Web: 19 de dezembro de 2016

http://rvq.sbq.org.br

\title{
Ethyl 2-diazoacetoacetate (CAS No. 2009-97-4)
}

Abstract: This paper shows a brief abstract of the synthesis and applications of ethyl 2diazoacetoacetate. This compound is considered an important reactant used as a synthetic precursor on various chemical transformations. The general method for their synthesis involves the direct diazo transfer reaction using different diazo group transfer reagent and basic conditions.

Keywords: Ethyl 2-diazoacetoacetate; synthesis; applications.

\section{Resumo}

Este trabalho faz um breve resumo das sínteses e aplicações do 2-diazoacetoacetato de etila. Este composto é considerado um importante reagente utilizado como precursor sintético em diversas transformações químicas. O método geral para a sua preparação envolve a reação de transferência direta do grupo diazo, utilizando diferentes tipos de reagentes de diazotação sob condições básicas.

Palavras-chave: 2-diazoacetoacetato de etila; síntese; aplicações.

* Universidade Federal Fluminense, Departamento de Química Orgânica, Programa de PósGraduação em Química, Outeiro de São João Batista, s/n , CEP 24020-141, Niterói-RJ, Brasil.

M fernanda lima@id.uff.br

DOI: $10.21577 / 1984-6835.20160141$ 


\section{2-Diazoacetoacetato de Etila (CAS No. 2009-97-4)}

\section{Fernanda Alves Lima*}

Universidade Federal Fluminense, Departamento de Química Orgânica, Programa de PósGraduação em Química, Outeiro de São João Batista, s/nº CEP 24020-141, Niterói-RJ, Brasil.

* fernanda lima@id.uff.br

Recebido em 1 de julho de 2016. Aceito para publicação em 16 de dezembro de 2016

\section{Histórico e Síntese}

2. Aplicações do 2-diazoacetoacetato de etila em síntese orgânica

\section{Conclusão}

\section{Histórico e Síntese}

Substâncias orgânicas contendo o grupo diazo vêm sendo transformadas ao longo dos últimos anos em inúmeros compostos químicos distintos via reações de transferência direta do grupo diazo em enaminas apropriadamente substituídas, ${ }^{1,2}$ ciclopropanação, ${ }^{3-5}$ formação de ilídeos ${ }^{6-8}$, inserção de ligação $X-H(X=C, O, N, S),{ }^{9}$ cicloadição 1,3-dipolar ${ }^{10-11}$, cicloadição $[3+2],{ }^{12,13}$ dentre outras ${ }^{14,15}$ (Figura 1 ).
Atualmente existe uma variedade de metodologias para a preparação de diazocompotos, destacando-se entre elas, as reações de: clivagem alcalina de $N$-alquil- $N$ nitroso derivados de sulfonamidas, carboxamidas, ureias e uretanas; ${ }^{15}$ diazotização de aminas alifáticas primárias; ${ }^{16,17}$ desidrogenação de hidrazonas; $^{18}$ tratamento básico de sulfonilhidrazonas ${ }^{19}$ e de transferência direta e indireta do grupo diazo para compostos carbonilados. $^{20,21}$ 
<smiles>[R]C(=O)C1CC(C(=O)OCC)=NN1</smiles>

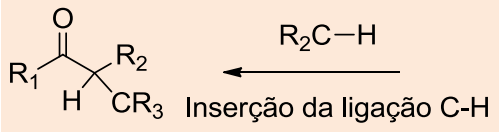
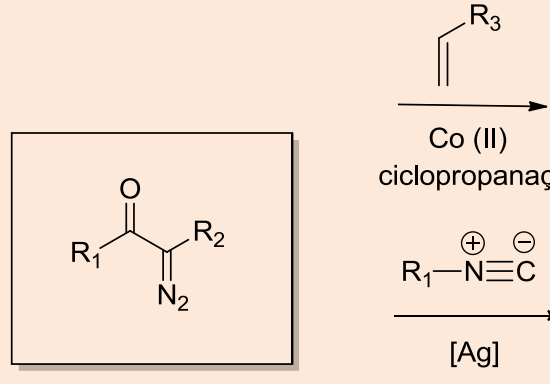<smiles></smiles>

ciclopropanação

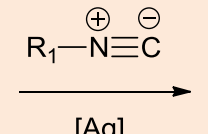

[Ag]

Cicloadição [3+2]<smiles>[R]c1cn([R])nn1</smiles>

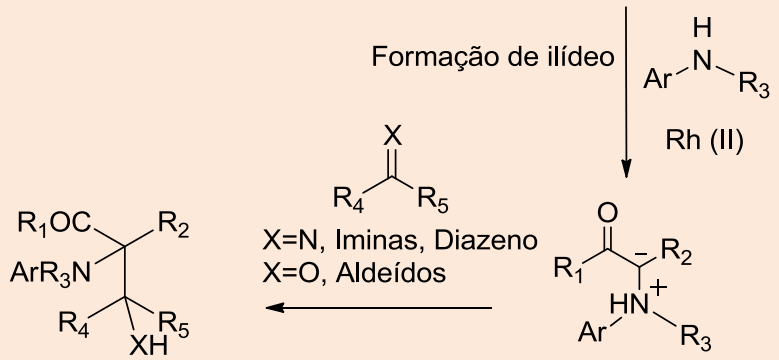

Figura 1. Aplicações sintéticas de compostos $\alpha$-diazocarbonílicos

Em especial, o 2-diazoacetoacetato de etila, um óleo de coloração amarela e de densidade $1,131 \mathrm{~g} / \mathrm{mL}\left(25^{\circ} \mathrm{C}\right)$ (Figura 2), vem sendo largamente utilizado como precursor sintético em diversas transformações químicas e está entre os reagentes mais estáveis que contém o grupamento diazo. ${ }^{15}$<smiles>CCOC(=O)C(=N)C(C)=O</smiles>

Figura 2. Estrutura do 2-diazoacetoacetato de etila

O método geral para a sua preparação envolve a reação de transferência direta do grupo diazo para a posição $\alpha$-metilênica do acetoacetato de etila, na presença de uma base orgânica, como a trietilamina $\left(\mathrm{Et}_{3} \mathrm{~N}\right)$, ou inorgânica, como o carbonato de potássio $\left(\mathrm{K}_{2} \mathrm{CO}_{3}\right)$ (Esquema 1$) .{ }^{22}$

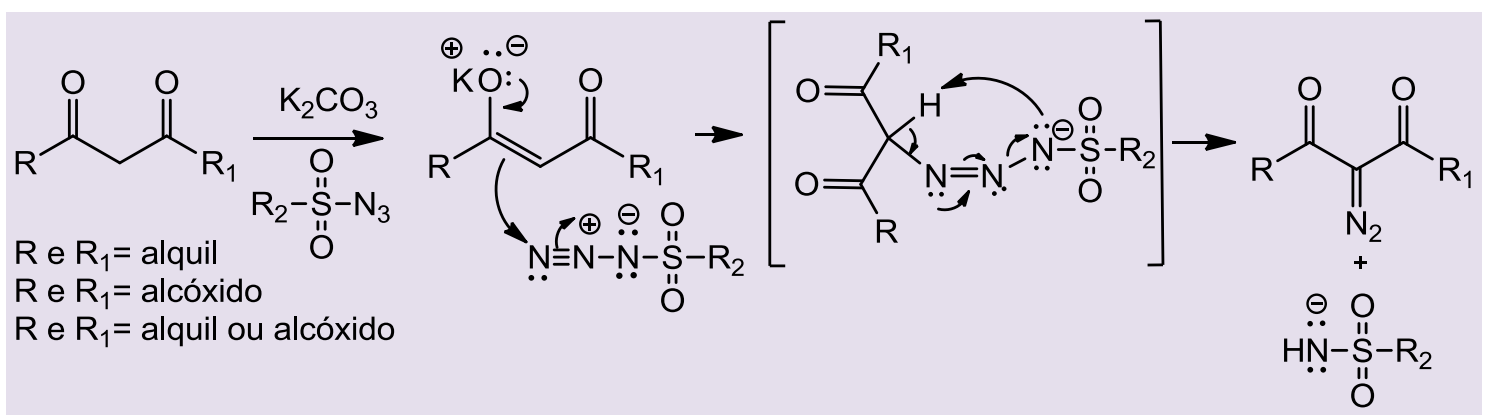

Esquema 1. Reação de transferência direta de diazo com sulfonilazidas como reagente de diazotação 
Nesta reação ocorre, inicialmente, a remoção do próton $\alpha$-carbonílico por uma base e, em seguida, há a transferência do grupo diazo pelos chamados reagentes de transferência de diazo ou de diazotação, como as sulfonilazidas, para a posição $\alpha$ metilênica da base conjugada do composto dicarbonilado. Após a liberação do ânion do grupamento sulfonamida há então a formação do composto 2-diazo-1,3- dicarbonilado.

Cabe destacar que os reagentes sulfonilazidas devem ser manipulados com cuidado, uma vez que espécies reativas como os sulfonilnitrenos podem ser formadas por decomposição térmica dos mesmos. ${ }^{22,23} \mathrm{Na}$ Figura 3 a seguir estão apresentados alguns exemplos de sulfonilazidas comumente usadas em reações de transferência direta do grupo diazo.

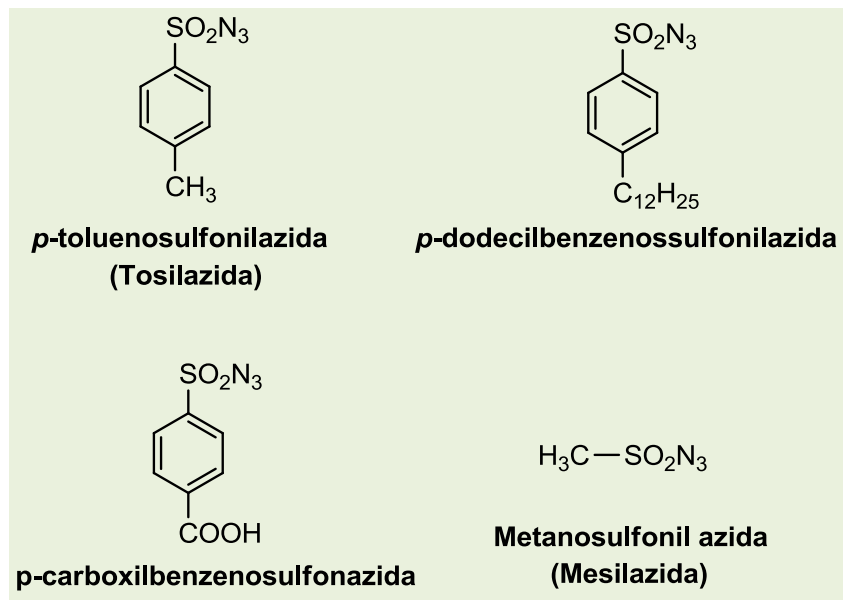

Figura 3. Reagentes de transferência de diazo

Por ser facilmente acessível, de fácil preparação e possuir alta eficiência em pequena escala, a $p$-toluenossulfonil azida (tosilazida) é o reagente empregado mais frequentemente. Entretanto, a sua utilização em reações de diazotação é limitada devido a vários fatores, tais como: i) difícil separação do composto $\alpha$-diazocarbonílico do subproduto sulfonamida por métodos cromatográficos convencionais; ii) potencial perigo de decomposição devido à combinação de fatores como alta sensibilidade ao impacto, alto calor de decomposição e baixa temperatura de ignição, sendo assim, explosivo sob várias condições reacionais. ${ }^{23}$

A $p$-dodecilbenzenossulfonilazida tem como vantagens o baixo calor de decomposição e a insensibilidade a altos impactos. Além disso, o subproduto da reação, a $p$-dodecilbenzenossulfonamida, não é sólido, facilitando a purificação dos produtos, no caso de síntese de diazocetonas sólidas. $^{23}$

A $p$-carboxilbenzenosulfonazida possui temperatura de ignição elevada e a presença do grupamento carboxílico facilita o isolamento de substâncias neutras. Entretanto, este reagente de transferência de diazo possui preço elevado e nas reações em que o mesmo é empregado necessita-se de dois equivalentes de base para cada equivalente de substrato, o que não é ideal para substâncias sensíveis à bases. ${ }^{23}$

A mesilazida tem como vantagens o baixo custo de preparação e a grande facilidade com que, tanto a mesilazida usada em excesso, quanto o subproduto da reação, a sulfonamida, podem ser removidos da mistura reacional através de extração ácidobase como, por exemplo, empregando-se solução aquosa de $\mathrm{NaOH} 10 \% .{ }^{23}$ 
O uso de argilas em substituição a bases orgânicas e inorgânicas foi relatado por Ferreira e colaboradores ${ }^{24}$, conforme Esquema 2 a seguir. Este protocolo permitiu que o 2-diazoacetoacetato de etila fosse sintetizado com bom rendimento, empregando-se argilas como suporte sólido para promover a reação sob condições heterogêneas. A fácil recuperação e reutilização do material de origem mineral tornam o método prático e de baixo custo.

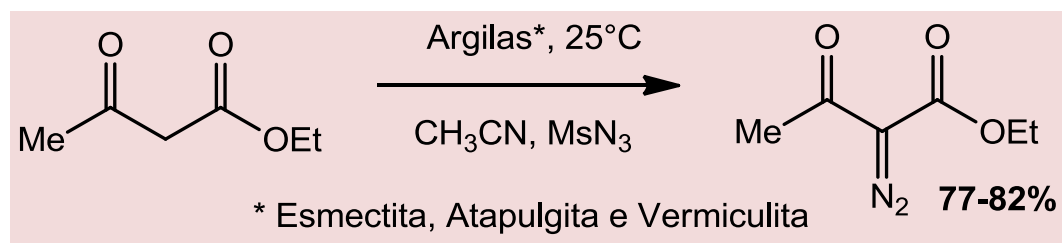

Esquema 2. Reação de transferência de diazo com o uso de argilas

Recentemente, Dutra e colaboradores ${ }^{25}$ ampliaram a metodologia de transferência do grupo diazo, com o uso de peneira molecular, obtendo o 2-diazoacetoacetato de etila com alto rendimento (Esquema 3).

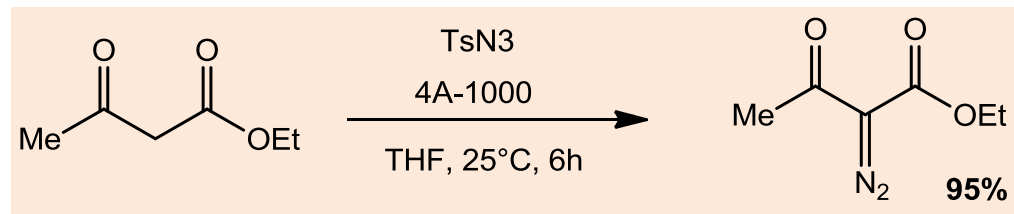

Esquema 3. Reação de transferência de diazo promovida por peneira molecular

A síntese do 2-diazoacetoacetato de etila empregando-se o reagente de transferência de diazo suportado em poliestireno, além fornecer melhores condições de segurança de trabalho, permite que este produto seja isolado facilmente na sua forma pura $^{26}$ (Esquema 4).<smiles>CCOC(=O)CC(C)=O</smiles>

Esquema 4. Reação de transferência de diazo utilizando-se benzenossulfonil azida suportada em poliestireno

A reação de diazotação do acetoacetato de etila com mesilazida na presença de líquidos iônicos, como [bmim][Br], levou à obtenção do 2-diazoacetoacetato de etila com alto rendimento ${ }^{27}$ (Esquema 5).

A síntese do 2-diazoacetoacetato de etila baseada na utilização de sais de 2-azido-1,3dimetil imidazolínio em substituição às sulfonilazidas comumente utilizadas foi proposta por M. Kitamuraet e colaboradores $^{28}$ (Esquema 6). Neste tipo de reação o composto 2-diazo-1,3-dicarbonílico é obtido em bom rendimento e seu isolamento é facilitado devido à alta solubilidade dos subprodutos em água. 
<smiles>CCOC(=O)CC(C)=O</smiles>

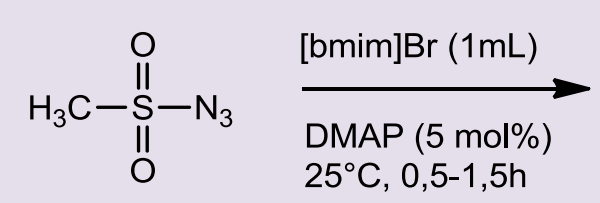<smiles>CCOC(=O)C(=N)C(C)=O</smiles>

Esquema 5. Reação de transferência de diazo catalisada por líquido iônico<smiles>CCOC(=O)CC(C)=O</smiles>

Esquema 6. Reação de transferência de diazo promovida por sal orgânico

O método de acoplamento $\mathrm{C}-\mathrm{C}$ catalisado por metais de transição também tem sido empregado para a funcionalização de diazocompostos. Um exemplo desta metodologia leva à síntese do 2- diazoacetoacetato de etila via acoplamento cruzado do diazoacetato de etila com iodeto de metila catalisado por sal de paládio, na presença de monóxido de carbono ${ }^{29,30}$ (Esquema 7).<smiles>CCOC(=O)C=[N+]=[Ni]</smiles>

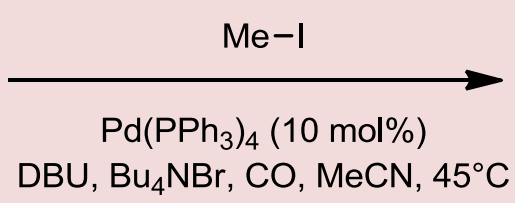<smiles>CCOC(=O)C(=N)C(C)=O</smiles>

Esquema 7. Reação de funcionalização do diazoacetato de etila via acoplamento cruzado

A seguir estão mostrados alguns exemplos de aplicações em síntese orgânica do 2diazoacetoacetato de etila.

\section{Aplicações do 2- diazoacetoacetato de etila em síntese orgânica}

1,2,3-Triazóis representam uma classe de compostos azólicos que exibem diferenciadas atividades biológicas, destacando-se entre elas antitumoral, ${ }^{31-33}$ antiviral, ${ }^{34-36}$ antimicrobiana, ${ }^{37}$ antifúngica, ${ }^{38}$ tuberculostática. ${ }^{39} \mathrm{Um}$ dos métodos gerais para a construção de anéis 1,2,3-triazólicos substituídos é a 1,5-eletrociclização intramolecular de compostos $\alpha$ diazocarbonílicos- $\beta$-substituídos. ${ }^{40-42} \quad$ Este método, também conhecido como eletrociclização do tipo $[2 \mathrm{~N}+1 \mathrm{~N}]$, consiste em combinar um reagente contendo dois átomos de nitrogênio com outro que possui apenas um átomo de nitrogênio, podendo se seguir duas vias distintas: a partir de cetonas ou ésteres $\beta$-amino- $\alpha, \beta$-insaturados, faz-se a reação de transferência de diazo (Via I) ou a partir de um composto $\alpha$-diazocarbonílico, forma-se, por sua reação com aminas, o intermediário $\alpha$-diazoimino (Via II). Nas duas vias, 0 intermediário $\alpha$-diazo- $\beta$-iminocarbonílico cicliza formando, então, os derivados de 1,2,3-triazóis (Esquema 8). Uma vantagem da Via II é a formação de apenas 
um regioisômero, uma vez que a reação de condensação é dirigida para o grupo formil ou ceto, que são mais eletrofílicos do que um grupo acil. ${ }^{43}$

Esta metodologia da construção de 1,2,3triazóis a partir de um composto $\alpha$ diazocarbonílico foi aplicada para a preparação de vários triazóis biologicamente ativos. Cunha e colaboradores ${ }^{44}$ relataram a síntese de uma série congênere de 1-arilsubstituídas-sulfonilamino-5-metil- $1 H$-[1,2,3]triazóis, explorando a reação de 1,5eletrociclização entre o 2-diazoacetoacetato de etila e diferentes sulfonilidrazidas (Esquema 9). Estas substâncias foram capazes de neutralizar a propriedade hemolítica do veneno da cobra Lachesis muta, sendo o derivado clorado o composto mais potente desta família de 1,2,3-triazóis.

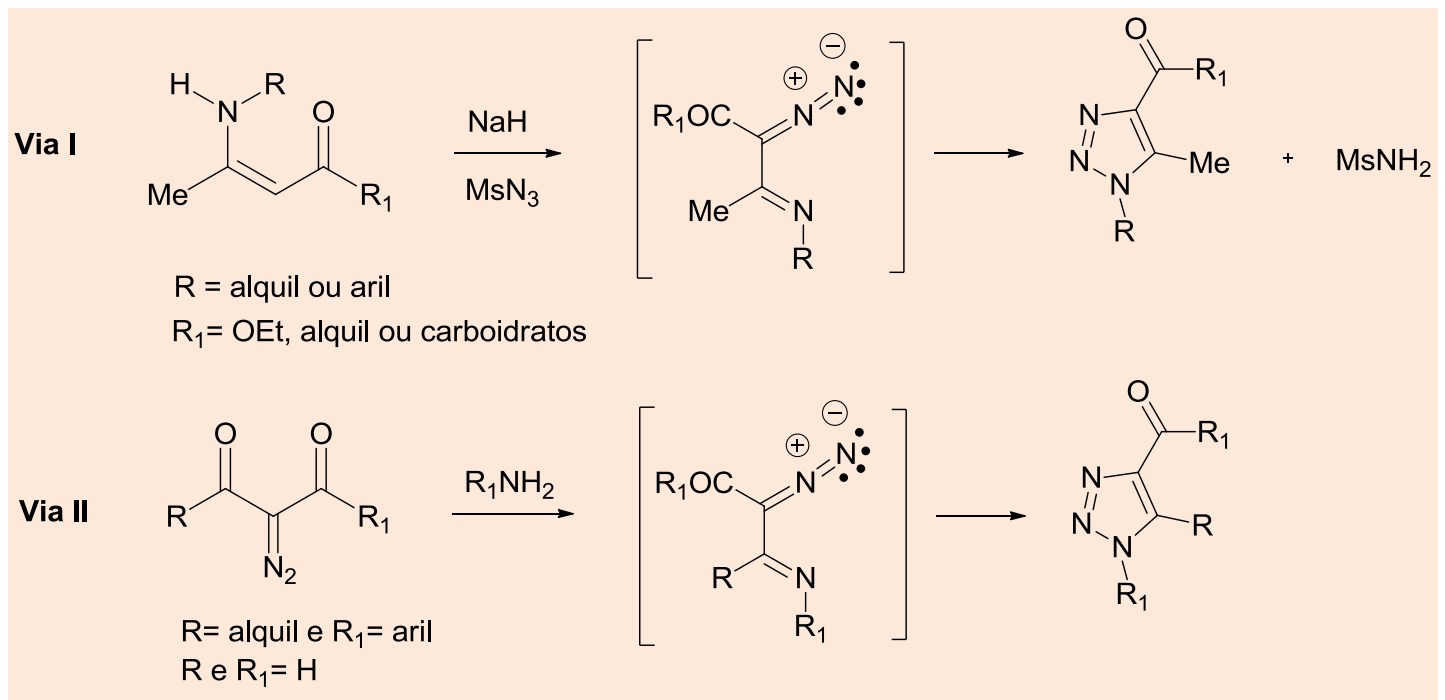

Esquema 8. Método para a síntese de 1,2,3-triazóis via intermediários $\alpha$-diazoiminas

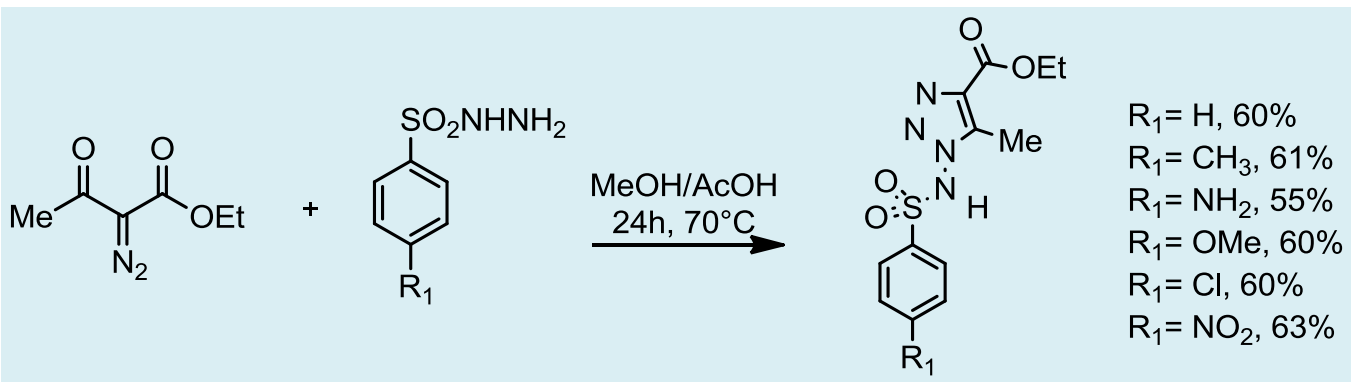

Esquema 9. Reação de 1,5-eletrociclização entre o 2-diazo acetoacetato de etila e diferentes sulfonilidrazidas

Ainda empregando-se a mesma metodologia, Cunha e colaboradores ${ }^{45}$ propuseram a reação do 2-diazoacetoacetato de etila com fenilidrazina ou cloridratos de fenilidrazina adequadamente substituídos, levando à formação de $\mathrm{N}$-amino-1,2,3-triazóis com bons rendimentos (Esquema 10). Estas substâncias e os derivados acilidrazidas formados reagindo-se os 4-carbetoxi-triazóis correspondentes com solução aquosa de hidrazina, apresentaram atividade antiviral quando avaliados frente à replicação do vírus Cantagalo. 
<smiles>CCOC(=O)C(=[N+]=[N-])C(C)=O</smiles>

ou<smiles>[R1]c1cccc(Nn2nnc(C(=O)OCC)c2C)c1</smiles>

$\mathrm{NH}_{2} \mathrm{NH}_{2} 80 \%$

$\mathrm{EtOH}$, refluxo

$\mathrm{R}_{1}=\mathrm{H}, 73 \%$

$\mathrm{R}_{1}=4-\mathrm{Cl}, 62 \%$

$\mathrm{R}_{1}=4-\mathrm{Br}, 62 \%$

$\mathrm{R}_{1}=2,5-\mathrm{Cl}, 56 \%$

$\mathrm{R}_{1}=4-\mathrm{F}, 40 \%$<smiles>Cc1c(C(=O)NN)nnn1Nc1ccc(Br)cc1</smiles>

$\mathrm{R}_{1}=\mathrm{H}, 56 \%$

$\mathrm{R}_{1}=4-\mathrm{Cl}, 58 \%$

$\mathrm{R}_{1}=4-\mathrm{Br}, 62 \%$

$\mathrm{R}_{1}=2,5-\mathrm{Cl}, 50 \%$

$\mathrm{R}_{1}=4-\mathrm{F}, 50 \%$

Esquema 10. Síntese de $\mathrm{N}$-aminotriazóis e acilidrazidas via 2-diazoacetoacetato de etila

Os análogos de acilidrazidas foram utilizados como intermediários para a obtenção de uma série de $\mathrm{N}$-Acilidrazonas bioativas (NAH) (Esquema 11), as quais foram testadas quanto ao seu perfil in vitro contra a agregação de plaquetas humanas, utilizando o ácido araquidónico, a adrenalina e o ADP como agonistas. Estas substâncias apresentaram atividade antiplaquetária significativa. ${ }^{46}$

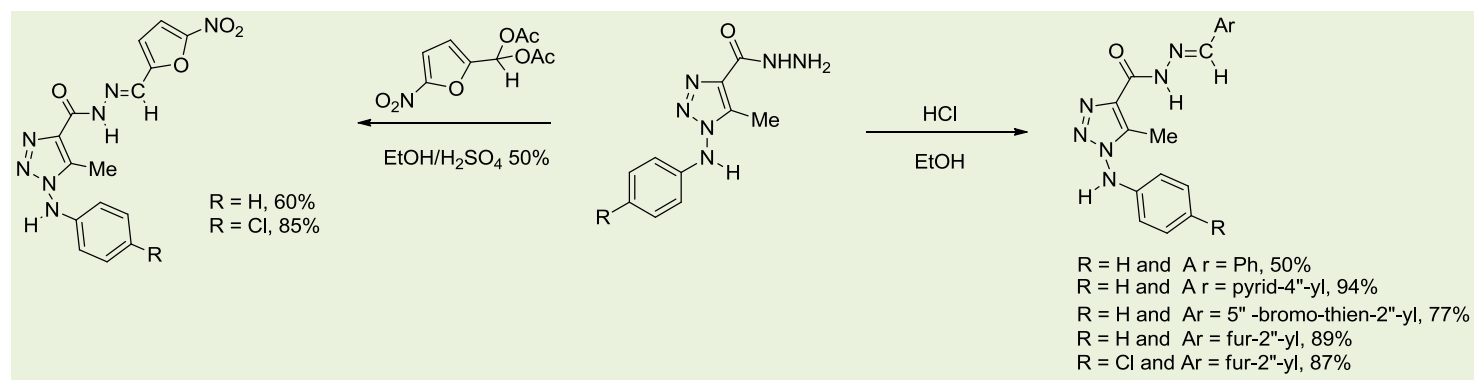

Esquema 11. Síntese de acilidrazonas triazólicas bioativas

Xia e colaboradores ${ }^{47}$ descreveram a síntese em um único pote de diidrofuranos tri-substituídos via reação de cicloadição
[3+2], catalisada por complexo de Ru (II), entre o 2-diazoacetoacetato de etila e diferentes olefinas (Esquema 12).

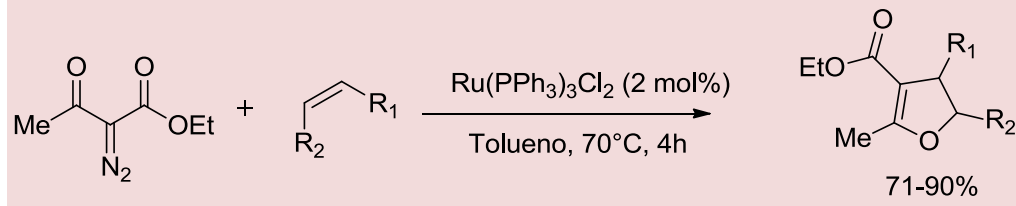

Esquema 12. Síntese de diidrofuranos a partir do 2-diazoacetoacetato de etila via cicloadição $[3+2]$

As reações de inserção do grupo $\mathrm{OH}$ (Equação I, Esquema 13) e de condensação (Equações II e III, Esquema 13) entre o 2diazoacetoacetato de etila e diferentes álcoois, nitrilas, aldeído e cetonas, catalisadas por complexos de Ru (II), levaram ao desenvolvimento de compostos de diferentes classes funcionais. ${ }^{48}$ 


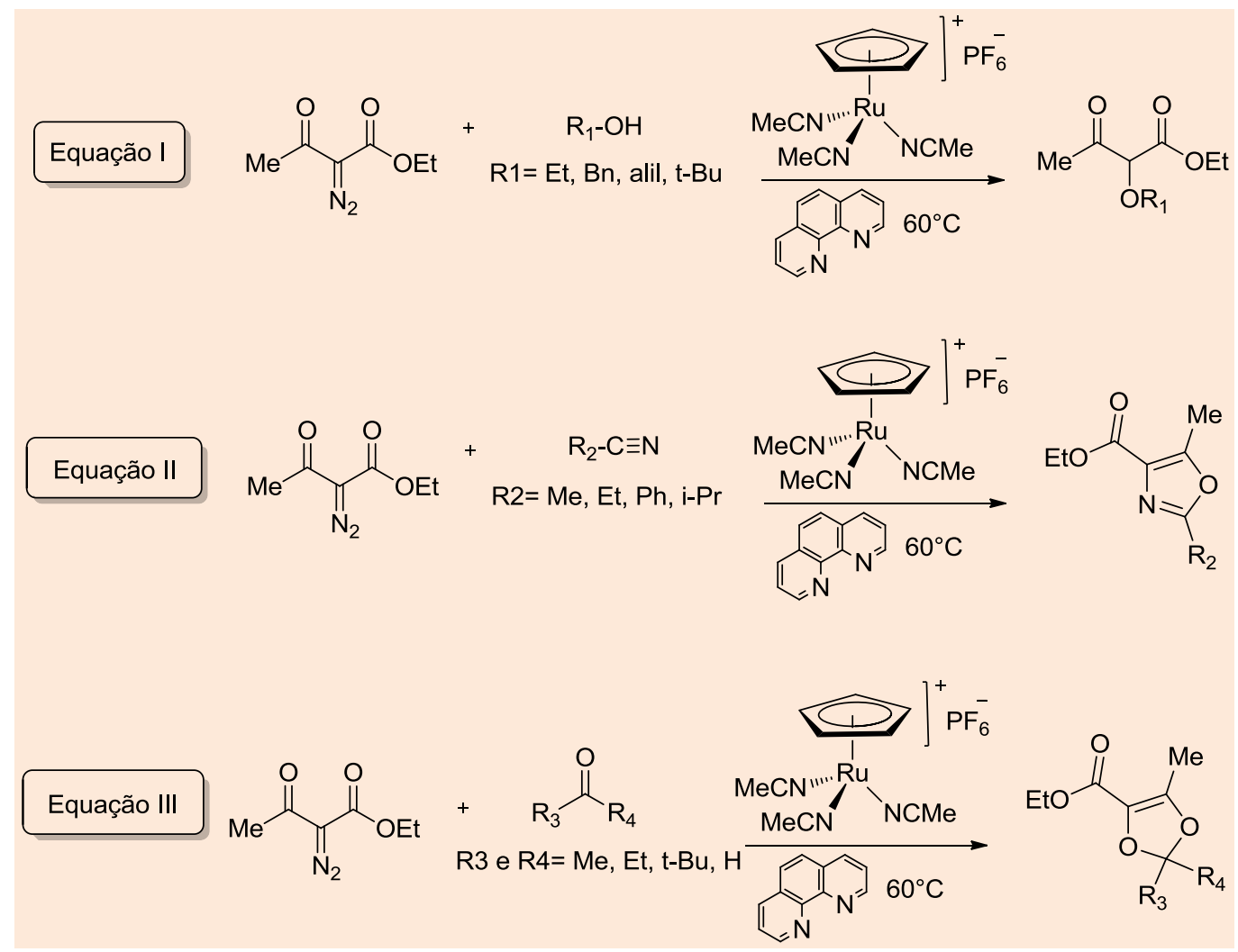

Esquema 13. Síntese de diversos compostos a partir do 2-diazoacetoacetato de etila

Em estudos de reações de compostos diazodicarbonilados, Muthusamy e colaboradores $^{49}$ realizaram a reação do 2diazoacetoacetato de etila com trifenilfosfina em meio aquoso obtendo o derivado hidrazônico com alto rendimento (Esquema 14).<smiles>CCOC(=O)C(=N)C(=O)C(C)=O</smiles>

Esquema 14. Síntese de hidrazona a partir do 2-diazoacetoacetato de etila

A síntese regiosseletiva de derivados furânicos tri-substituídos via ciclização radicalar intermolecular, catalisada por Cobalto, foi desenvolvida por Cui e colaboradores ${ }^{50}$. Reagindo fenilacetileno com 2-diazoacetoacetato de etila obtiveram o derivado furânico correspondente com $73 \%$ de rendimento (Esquema 15). 
<smiles>CCOC(=O)C(N)=O</smiles><smiles></smiles>
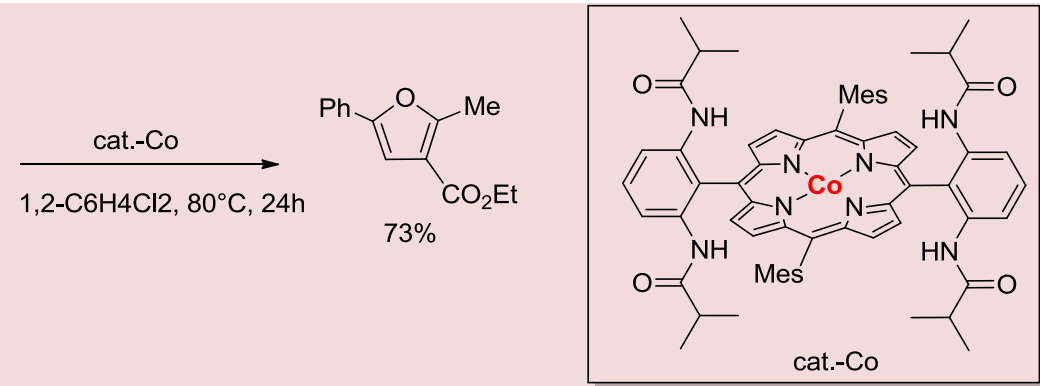

Esquema 15. Síntese de furano tri-substituído a partir do 2-diazoacetoacetato de etila, catalisada por cobalto

Ferreira e colaboradores ${ }^{51}$ relataram a decomposição catalisada por tetraacetato de di-ródio do 2-diazoacetoacetato de etila na presença de éter butil vinílico para a síntese do intermediário acetato de 5-butoxi-2-metil4,5-diidrofurano-3-carboxilato de etila. A reação deste intermediário, com excesso de amina primária na presença de ácido acético glacial, proporcionou a síntese do 4-acil-2metil-1H-pirrol correspondente, em um único pote (Esquema 16).

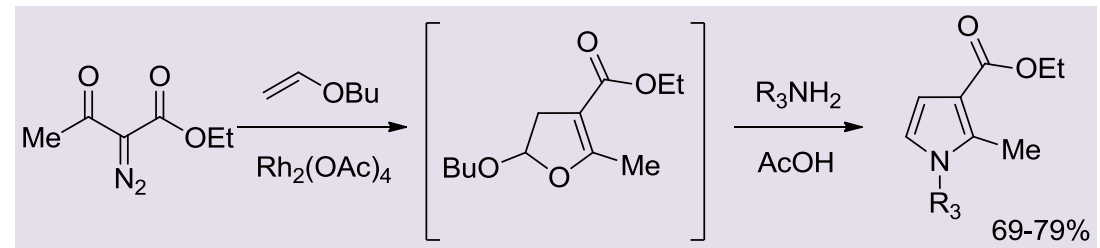

Esquema 16. Síntese de pirrol a partir da decomposição do 2-diazoacetoacetato de etila catalisada por ródio

Ye et $a .^{52}$ relataram o acoplamento cruzado entre diferentes iodetos aromáticos e 2-diazoacetoacetato de etila, catalisado por paládio, para a obtenção dos arildiazoacetatos com bons rendimentos (Esquema 17).
A reação de condensação do oxetano com 2-diazoacetoacetato de etila, catalisada por ródio (II), forneceu exclusivamente um tipo raro de macrociclopoliéter funcionalizado de 15 membros $^{53}$ (Esquema 18).

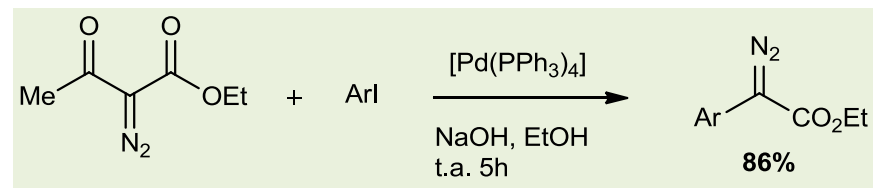

Esquema 17. Síntese de arildiazoacetatos a partir da reação de 2-diazoacetoacetato de etila com iodetos aromáticos, catalisada por paládio 
<smiles>CCOC(=O)C(=N)C(C)=O</smiles><smiles>CCOC(=O)/C(OCCCOCCO)=C(\C)OCC</smiles>

Esquema 18. Síntese de um macrociclopoliéter funcionalizado de 15 membros a partir do 2diazoacetoacetato de etila

O 2-diazoacetoacetato de etila quando submetido à reação com a $\alpha$ isocianoacetamida, discriminada no Esquema 19, forneceu o amino oxazoltrisubstituído com bom rendimento. ${ }^{54} \mathrm{Em}$ termos de economia atômica, esta síntese permite uma maximização da incorporação dos átomos presentes nas matérias-primas, tendo em vista que apenas uma molécula de nitrogênio é liberada durante toda a transformação química (Esquema 19).

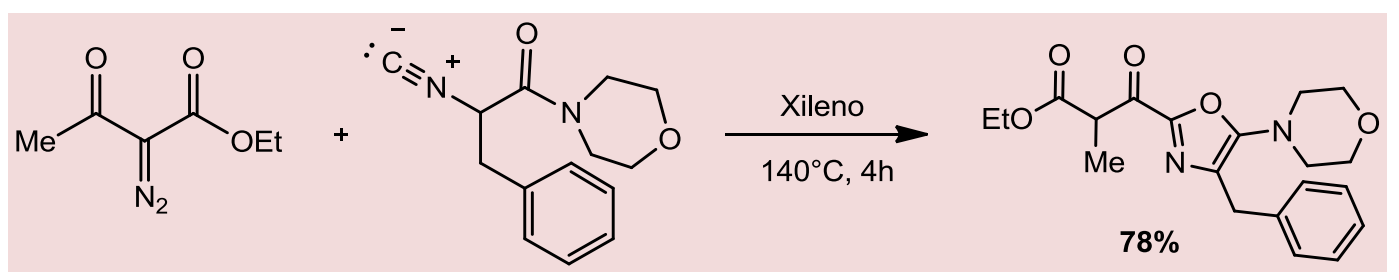

Esquema 19. Síntese de amino oxazol trisubstituído a partir do 2-diazoacetoacetato de etila

\section{Conclusão}

Compostos $\alpha$-diazocarbonílicos tem atraído a atenção dos químicos orgânicos devido a sua alta versatilidade sintética. 0 2diazoacetoacetato de etila é um dos representantes mais estáveis desta classe e possui extensa aplicabilidade, participando como precursor na síntese de moléculas com diferentes propostas, dentre elas heterociclos com atividades biológicas e macrociclos funcionalizados.

Nessa revisão foram expostas metodologias atuais para a preparação do 2diazoacetoacetato de etila, utilizando diferentes reagentes de diazotação e meios reacionais, como também reações envolvendo este importante precursor na síntese de compostos de diversas classes funcionais, com destaque para a síntese de heterociclos.

\section{Agradecimentos}

Às agências CAPES e CNPq pelo suporte financeiro.

\section{Referências Bibliográficas}

${ }^{1}$ Romeiro, G. A.; Pereira, L. O. R.; Souza, M. C. B. V.; Ferreira, V. F.; Cunha, A. C. A New and Efficient Procedure for Preparing 1,2,3triazoles. Tetrahedron Letters 1997, 38, 5103. [CrossRef]

${ }^{2}$ da Silva, F. C.; de Souza, M. C. B. V.; Frugulhetti, I. I. P.; Castro, H. C.; Souza, S. L. O.; de Souza, T. M. L.; Rodrigues, D. Q.; Souza, A. M. T.; Abreu, P. A.; Passamani, F.; Rodrigues, C. R.; Ferreira, V. F. Synthesis, HIVRT inhibitory activity and SAR of 1-benzyl-1H1,2,3-triazole derivatives of carbohydrates. European Journal of Medicinal Chemistry 2009, 44, 373. [CrossRef] [PubMed] 
${ }^{3}$ Kissane, M.; Maguire, A. R. Asymmetric 1,3dipolar cycloadditions of acrylamides. Chemical Society Reviews 2010, 39, 845. [CrossRef] [PubMed]

${ }^{4}$ Dzik, W. I.; Xu, X.; Zhang, P.; Reek, J. N. H.; de Bruin, B. Carbene Radicals' in Coll (por)-C atalyzed Olefin Cyclopropanation. Journal of the American Chemical Society 2010, 132, 10891. [CrossRef] [PubMed]

${ }^{5}$ Cui, X.; Xu, X.; Lu, H.; Zhu, S.; Wojtas, L.; Zhang, $X$. P. Enantioselective Cyclopropenation of Alkynes with Acceptor/Acceptor-Substituted Diazo Reagents via Co(II)-Based Metalloradical Catalysis. Journal of the American Chemical Society 2011, 133, 3304. [CrossRef] [PubMed]

${ }^{6}$ Muthusamy, S.; Srinivasan, P. $\mathrm{PPh}_{3}-$ mediated reactions of diazoimides in water: a facile synthesis of fused triazine derivatives. Tetrahedron Letters 2009, 50, 1331. [CrossRef]

${ }^{7}$ Medvedev, J. J.; Nikolaev, V. A. Recent advances in the chemistry of Rh carbenoids: multicomponent reactions of diazocarbonyl compounds. Russian Chemical Reviews 2015, 84, 737. [CrossRef]

${ }^{8}$ Li, Z.; Parr, B. T.; Davies, H. M. L. Highly Stereoselective $\mathrm{C}-\mathrm{C}$ Bond Formation by Rhodium-Catalyzed Tandem Ylide Formation/[2,3]-Sigmatropic Rearrangement between Donor/Acceptor Carbenoid sand Chiral Allylic Alcohols. Journal of the American Chemical Society 2012, 134, 10942. [CrossRef]

${ }^{9}$ Austeri, M.; Rix, D.; Zeghida, W.; Lacour, J. CpRu-Catalyzed O-H Insertion and Condensation Reactions of $\alpha$-Diazocarbonyl Compounds. Organic Letters 2011, 13, 1394. [CrossRef] [PubMed]

${ }^{10}$ Aronoff, M. R.; Gold, B.; Raines, R. T. 1,3Dipolar Cycloadditions of DiazoCompounds in the Presence of Azides. Organic Letters 2016, 18, 1538. [CrossRef] [PubMed]

${ }^{11}$ Liu, Y.; Wang, Z.; Shi, J.; Chen, B.; Zhao, Z.; Chen, Z. NHC-Ag(I)-Catalyzed ThreeComponent 1,3-Dipolar Cycloaddition To Provide Polysubstituted Dihydro-
/Tetrahydrofurans. The Journal of Organic Chemistry 2015, 80, 12733. [CrossRef] [PubMed]

${ }^{12}$ Xia, L.; Lee, Y. R. Efficient One-Pot Synthesis of Multi-Substituted Dihydrofurans by Ruthenium(II)-Catalyzed [3+2] cycloaddition of Cyclic or Acyclic Diazodicarbonyl Compounds with Olefins. Advanced Synthesis and Catalysis 2013, 355, 2361. [CrossRef]

${ }^{13}$ Chiara, J. L.; Suárez, J. R. Synthesis of aDiazo Carbonyl Compounds with the ShelfStable Diazo Transfer Reagent nonafluorobutanesulfonyl Azide. Advanced Synthesis and Catalysis 2011, 353, 575. [CrossRef]

${ }^{14}$ Padwa, A.; Sá, M. M. Reações de inserção intramolecular de diazo compostos polifuncionais catalisadas por ródio(II): síntese de oxetan-3-ona-2-carboxilato e outros heterociclos funcionalizados. Química Nova 1999, 22, 815. [CrossRef]

${ }^{15}$ Ferreira, V. F.; Pereira, L. O. R.; de Souza, M. C. B. V.; Cunha, A. C. Compostos $\alpha$-diazo carbonílicos: uma estratégia atraente para a síntese orgânica. Química Nova 2001, 24, 540. [CrossRef]

${ }^{16}$ Schroen, M.; Brase, S. Polymer-Bound Diazonium Salts For the Synthesis of Diazoacetic Esters. Tetrahedron 2005, 61, 12186. [CrossRef]

${ }^{17}$ Dutra, L. G.; Tese de Doutorado, Universidade Federal de Santa Catarina, 2015.

18 Javed, M. I.; Brewer, M. Diazo Preparation via Dehydrogenation of Hydrazones with "Activated" DMSO. Organic Letters 2007, 9, 1789. [CrossRef] [PubMed]

${ }^{19}$ Khalili, G. A Novel Synthesis of Arylsulfonyl Hydrazine Derivatives Via the Reaction of Arylsulfonyl Hydrazone Salts and Hydrazonoyl Chlorides. Phosphorus, Sulfur, and Silicon and the Related Elements 2014, 189, 1882. [CrossRef]

${ }^{20}$ Maas, G. New Syntheses of Diazo Compounds. Angewandte Chemie 
International Edition 2009, 48, 8186. [CrossRef] [PubMed]

${ }^{21}$ Dutra, L. G.; Saibert, S.; Vicentini, D. S.; Sá, M. M. Diazo transfer reaction to 1,3dicarbonyl compounds with sulfonylazides catalyzed by molecular sieves. Journal of Molecular Catalysis A: Chemical 2014, 386, 35. [CrossRef]

22 Dias, F. R. F. Ethyl 2-Diazoacetoacetate. Synlett 2013, 24, 397. [CrossRef]

${ }^{23}$ Cunha, A. C.; Tese de doutorado, Universidade Federal Fluminense, 1999.

${ }^{24}$ Rianelli, R. S.; de Souza, M. C. B. V.; Ferreira, V. F. Mild Diazo Transfer Reaction Catalyzed by Modified Clays. Synthetic Communications 2004, 34, 951. [CrossRef]

${ }^{25}$ Dutra, L. G.; Saibert, S.; Vicentini, D. S.; Sá, M. M. Diazo transfer reaction to 1,3dicarbonyl compounds with sulfonylazides catalyzed by molecular sieves. Journal of Molecular Catalysis A: Chemical 2014, 386, 35. [CrossRef]

${ }^{26}$ Green, G. M.; Peet, N. P.; Metz, W. A. Polystyrene-Supported Benzenesulfonyl Azide: A Diazo Transfer Reagent That Is Both Efficient and Safe. The Journal of Organic Chemistry 2001, 66, 2509. [CrossRef] [PubMed]

${ }^{27}$ Ramachary, D. B.; Narayana, V. V; Ramakumar, K. Direct ionic liquid promoted organocatalyzed diazo-transfer reactions: diversity-oriented synthesis of diazocompounds. Tetrahedron Letters 2008, 49, 2704. [CrossRef]

${ }^{28}$ Kitamura, M.; Tashiro, N.; Miyagawa, S.; Okauchi, T. 2-Azido-1,3dimethylimidazolinium Salts: Efficient DiazoTransfer Reagents for 1,3-Dicarbonyl Compounds. Synthesis 2011, 7, 1037. [CrossRef]

${ }^{29}$ Peng, C.; Cheng, J.; Wang, J. PalladiumCatalyzed Cross-Coupling of Aryl or Vinyl lodides with Ethyl Diazoacetate. Journal of the American Chemical Society 2008, 129, 8708. [CrossRef] [PubMed]

${ }^{30}$ Ye, F.; Wang, C.; Zhang, Y.; Wang, J. Synthesis of Aryldiazoacetates through
Palladium(0)-Catalyzed Deacylative CrossCoupling of Aryl lodides with Acyldiazoacetates. Angewandte Chemie International Edition 2014, 53, 11625. [CrossRef] [PubMed]

${ }^{31}$ Kallander, L. S.; Lu, Q.; Chen, W.; Tomaszek, T.; Yang, G.; Tew, D.; Meek, T. D.; Hofmann, G. A.; Schulz-Pritchard, C. K.; Smith, W. W.; Janson, C. A.; Ryan, M. D.; Zhang, G. F.; Johanson, K. O.; Kirkpatrick, R. B.; Ho, T. F.; Fisher, P. W.; Mattern, M. R.; Johnson, R. K.; Hansbury, M. J.; Winkler, J. D.; Ward, K. W.; Veber, D. F.; Thompson, S. K. 4Aryl-1,2,3-triazole: A novel template for a reversible methionine aminopeptidase inhibitor. Journal of Medicinal Chemistry 2005, 48, 5644. [CrossRef] [PubMed]

${ }^{32}$ Cheng, Z.; Li, W.; He, F.; Zhou, J.; Zhu, X. Synthesis and biological evaluation of 4-aryl5-cyano-2H-1,2,3-triazoles as inhibitor of HER2 tyrosinekinase. Bioorganic \& Medicinal Chemistry 2007, 15, 1533. [CrossRef] [PubMed]

${ }^{33}$ Guo, L.; Li, Z.; Wang, H.; Ye, C.; Zhang, D. Carboxyamido-triazole inhibits proliferation of human breast cancer cells via $\mathrm{G}(2) / \mathrm{M}$ cell cycle arrest and apoptosis. European Journal of Pharmacology 2006, 538, 15. [CrossRef] [PubMed]

${ }^{34}$ Telekar, R. R.; Wightman, R. H. Synthesis of some pyrrolo[2,3-d]pyrimidine and 1,2,3triazole isonnucleosides. Tetrahedron 1997, 53, 3831. [CrossRef]

${ }^{35}$ Xia, Y.; Fan, Z.; Yao, J.; Liao, Q.; Li, W.; Qu, F.; Peng, L. Discovery of bitriazolyl compounds as novel antiviral candidates for combating the tobacco mosaic virus. Bioorganic \& Medicinal Chemistry Letters 2006, 16, 2693. [CrossRef] [PubMed]

${ }^{36}$ da Silva, F. C.; Tese de doutorado, Universidade Federal Fluminense, 2007.

${ }^{37}$ Genin, M. J.; Allwine, D. A.; Anderson, D. J.; Barbachyn, M. R.; Emmert, D. E.; Garmon, S. A.; Graber, D. R.; Grega, K. C.; Hester, J. B.; Hutchinson, D. K.; Morris, J.; Reischer, R. J.; Ford, C. W.; Zurenko, G. E.; Hamel, J. C.; Schaadt, R. D.; Stapert, D.; Yagi, B. H. Substituent effects on the antibacterial 
activity of nitrogen-carbon-linked (azolylphenyl)oxazolidinones with expanded activity against the fastidious gram-negative organisms Haemophilus influenzae and Moraxella catarrhalis. Journal of Medicinal Chemistry 2000, 43, 953. [CrossRef] [PubMed]

${ }^{38}$ Pore, V. S.; Aher, N. G.; Kumar, M.; Shukla, P. K. Design and synthesis of fluconazole/bile acid conjugate using click reaction. Tetrahedron 2006, 62, 11178. [CrossRef]

${ }^{39}$ Costa, M. S.; Boechat, N.; Rangel, E. A.; da Silva, F. C.; de Souza, A. M.; Rodrigues, C. R.; Castro, H. C.; Junior, I. N.; Lourenço, M. C.; Wardell, S. M.; Ferreira, V. F. Synthesis, tuberculosis inhibitory activity, and SAR study of $\quad N$-substituted-phenyl-1,2,3-triazole derivatives. Bioorganic \& Medicinal Chemistry 2006, 14, 8644. [CrossRef] [PubMed]

${ }^{40}$ Melo, J. O. F.; Donnici, C. L.; Augusti, R.; Ferreira, V. F.; Souza, M. C. B. V.; Ferreira, M. L. G.; Cunha, A. C. 1,2,3-triazolic heterocycles: history, preparations, applications and pharmacological activities. Química Nova 2006, 29, 569. [CrossRef]

${ }^{41}$ Krivopalov, V. P.; Shkurko, O. P. 1,2,3Triazole and its derivatives: Development of methods for the formation of the triazole ring. Russian Chemical Reviews 2005, 74, 339. [CrossRef]

${ }^{42}$ Zhang, L.; Chen, X.; Xue, P.; Sun, H. H. Y; Williams, I. A. D.; Sharpless, K. B.; Fokin, V. V.; Jia, G. Ruthenium-Catalyzed Cycloaddition of Alkynes and Organic Azides. Journal of the American Chemical Society 2005, 127, 15998. [CrossRef] [PubMed]

${ }^{43}$ Jordão, A. K.; Ferreira, V. F.; Souza, T. M. L.; Faria, G. G. S.; Machado, V.; Abrantes, J. L.; de Souza, M. C. B. V.; Cunha, A. C. Synthesis and anti-HSV-1 activity of new 1,2,3-triazole derivatives. Bioorganic \& Medicinal Chemistry 2011, 19, 1860. [CrossRef] [PubMed]

${ }^{44}$ Campos, V. R.; Abreu, P. A.; Castro, H. C.; Rodrigues, C. R.; Jordão, A. K.; Ferreira, V. F.; de Souza, M. C.; Santos, F. C.; Moura, L. A.;
Domingos, T. S.; Carvalho, C.; Sanchez, E. F.; Fuly, A. L.; Cunha, A. C. Synthesis, biological, and theoretical evaluations of new 1,2,3triazoles against the hemolytic profile of the Lachesis muta snake venom. Bioorganic \& Medicinal Chemistry 2009, 17, 7429. [CrossRef] [PubMed]

${ }^{45}$ Jordão, A. F.; Ferreira, V. F.; Souza, T. M. L; Faria, G. G. S.; Machado, V. Abrantes, J. L.; de Souza, M. C. B. V.; Cunha, A. C. Antiviral evaluation of $\mathrm{N}$-amino-1,2,3-triazoles against Cantagalo virus replication in cell culture. European Journal of Medicinal Chemistry 2009, 44, 3777. [CrossRef] [PubMed]

${ }^{46}$ Jordão, A. K.; Ferreira, V. F.; Lima, E. S.; de Souza, M. C. B. V.; Carlos, E. C. L.; Castro, H. C.; Geraldo, R. B.; Rodrigues, C. R.; Almeida, M. C. B.; Cunha, A. C. Synthesis, antiplatelet and in silico evaluations of novel $\mathrm{N}$ substituted-phenylamino-5-methyl-1H-1,2,3triazole-4-carbohydrazides. Bioorganic \& Medicinal Chemistry 2009, 17, 3713. [CrossRef] [PubMed]

${ }^{47}$ Xia, L.; Lee, Y. R., Efficient One-Pot Synthesis of Multi-Substituted Dihydrofurans by Ruthenium(II)-Catalyzed [3+2] cycloaddition of Cyclic or Acyclic Diazodicarbonyl Compounds with Olefins. Advanced Synthesis and Catalysis 2013, 355, 2361. [CrossRef]

${ }^{48}$ Austeri, M.; Rix, D.; Zeghida, W.; Lacour, J.; CpRu-Catalyzed $\mathrm{O}-\mathrm{H}$ Insertion and Condensation Reactions of $\alpha$-Diazocarbonyl Compounds. Organic Letters 2011, 13, 1394. [CrossRef] [PubMed]

${ }^{49}$ Muthusamy, S.; Srinivasan, P. $\mathrm{PPh}_{3}{ }^{-}$ mediated reactions of diazoimides in water: $a$ facile synthesis of fused triazine derivatives. Tetrahedron Letters 2009, 50, 1331. [CrossRef]

${ }^{50}$ Cui, X.; Xu, X.; Wojtas, L.; Kim, M. M.; Zhang, $X$. P. Regioselective Synthesis of Multisubstituted Furans via Metalloradical Cyclization of Alkynes with $\alpha$-Diazocarbonyls: Construction of Functionalized $\alpha$-Oligofurans. Journal of the 
American Chemical Society 2012, 134, 19981. [CrossRef] [PubMed]

${ }^{51}$ da Silva, F. C.; Fonseca, M. G.; Rianelli, R. S.; Cunha, A. C.; de Souza, M. C. B. V.; Ferreira, V. F. One-pot preparation of substituted pyrroles from $\alpha$-diazocarbonyl compounds. Beilstein Journal of Organic Chemistry 2008, 4, 1. [CrossRef] [PubMed]

${ }^{52}$ Ye, F.; Wang, C.; Zhang, Y.; Wang, J. Synthesis of Aryldiazoacetates through Palladium(0)-Catalyzed Deacylative CrossCoupling of Aryl lodides with Acyldiazoacetates. Angewandte Chemie
International Edition 2014, 53, 11625. [CrossRef] [PubMed]

${ }^{53}$ Rix, D.; Garrido, R. B.; Zeghida, W.; Besnard, C.; Lacour, J. Macrocyclization of Oxetane Building Blocks with Diazocarbonyl Derivatives under Rhodium(II) Catalysis. Angewandte Chemie International Edition 2011, 50, 7308. [CrossRef] [PubMed]

${ }^{54} \mathrm{Wu}$, J.; Chen, W.; Hu, M.; Zou, H.; Yu, Y.; Synthesis of Polysubstituted 5-Aminooxazoles from $\alpha$ - Diazocarbonyl Esters and $\alpha$ Isocyanoacetamides. Organic Letters 2010, 12, 616. [CrossRef] [PubMed]

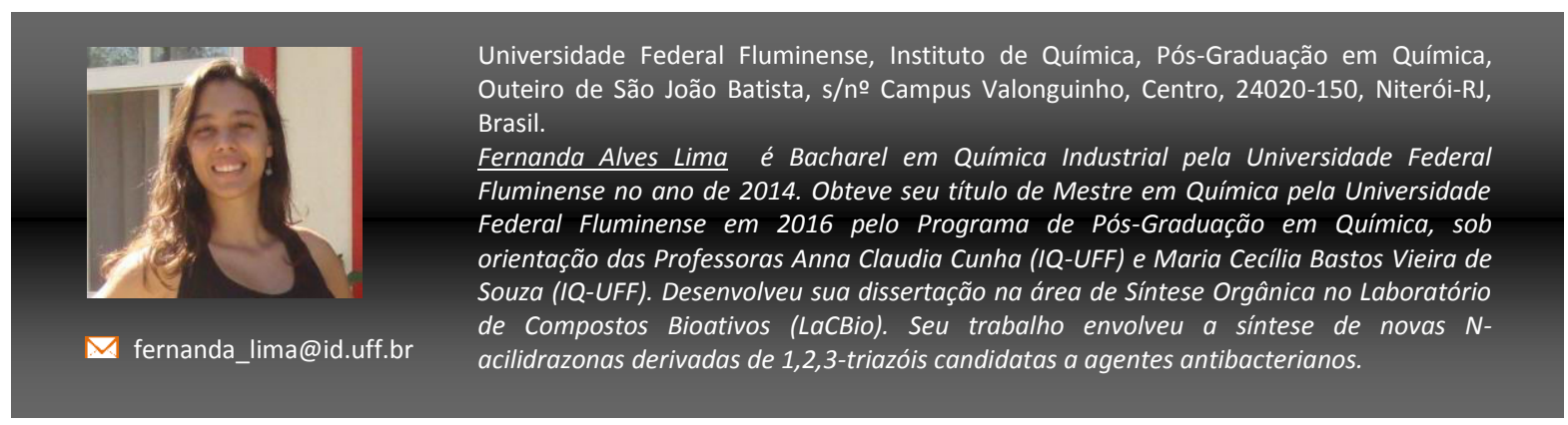

